\title{
ESTIMATE OF PARTICLE DENSITIES AND COLLISION DANGER FOR SPACECRAFT MOVING THROUGH THE ASTEROID BELT*
}

\author{
DONALD J. KESSLER \\ NASA Manned Spacecraft Center
}

The present lack of exact information about the distributions of asteroids and asteroidal meteoroids causes the largest uncertainty in the description of the interplanetary meteoroid environment between the orbits of Mars and Jupiter. Observed asteroids are inferred to have diameters from a few kilometers to a few hundred kilometers. The presence of these larger bodies suggests the presence of smaller, unobservable bodies. When asteroids collide, fragments are produced that eventually collide with other fragments. Because of this continuous collision process, much smaller asteroids most probably exist. Such asteroidal meteoroids, if present in sufficient number, could pose considerable danger to spacecraft.

\section{ASTEROID MASS DISTRIBUTION}

Various methods have been used to predict the number of smaller bodies in the asteroid belt. These methods include estimates of the mass distribution that are inferred from lunar and Martian crater distributions (Baldwin, 1964; Hartmann, 1965, 1968; Marcus, 1966, 1968), meteorite finds (Brown, 1960; Hawkins, 1964), and theoretical and experimental studies of rock crushing laws (Dohnanyi, 1969; Hawkins, 1960; Piotrowski, 1953). The number of smaller asteroids can also be estimated by the trend set by the larger asteroids. Most analyses indicate that the number of asteroids of mass $m$ and larger (the cumulative mass distribution) varies as $m^{-\alpha}$, where $\alpha$ is a constant. In extrapolating from the distributions for larger asteroids, a greater value for $\alpha$ indicates a larger number of smaller asteroids.

Several difficulties arise from inferring asteroid influx rates from lunar and Martian crater counts:

(1) The ages of the impacted surfaces are unknown.

(2) Controversy still exists about whether the craters were formed by asteroids, comet nuclei, secondary ejecta, or volcanism.

*The full text of this paper appears in NASA SP-8038, Oct. 1970. 
(3) The surface features of craters are eroded (by smaller meteoroids on the Moon or by atmospheric wind on Mars), which causes the smaller craters to disappear more rapidly than the larger craters.

(4) A surface can become saturated so that larger craters will obliterate a significant number of smaller craters. When this occurs, the number of impacting particles cannot be determined, and the original distribution is difficult to determine. Saturation appears to have taken place on the surface of Mars and in the lunar highlands (Marcus, 1966, 1968).

If meteorites are assumed to be of asteroidal origin, problems still exist in relating a meteorite mass to its original mass, because of ablation and fragmentation. Hawkins (1964) deduced the mass distribution of stony and iron meteorites and predicted their cumulative mass distribution in space to vary as $m^{-1}$ and $m^{-0.7}$, respectively. Brown (1960), on the other hand, found both stony and iron meteorites to vary as $m^{-0.77}$.

Several investigators have attempted to predict the number of smaller asteroids by theoretical and experimental studies of the effects of collisions between rocks. Piotrowski (1953) found that under certain restrictive conditions, erosion and breakup of asteroids would lead to a cumulative mass distribution that varies as $m^{-2 / 3}$. Hawkins (1960), however, pointed out that as terrestrial rocks are crushed, the value of $\alpha$ increases and approaches -1 . Dohnanyi (1969) used experimental results of hypervelocity impacts to determine a rock crushing law for the asteroids and their debris. He found that a steady-state solution exists if the cumulative mass distribution varies as $m^{-0.84}$. Dohnanyi also pointed out that his results are consistent with the observed asteroids of Kuiper et al. (1958).

Kessler (1969) used the individual orbits and absolute magnitudes of asteroids given in the 1967 Ephemeris volume to obtain the spatial density (number density) of asteroids as a function of absolute magnitude at various positions in space (every $0.1 \mathrm{AU}$ between 1.0 and $4.5 \mathrm{AU}$, and every $45^{\circ}$ of heliocentric longitude). The results for 1.5, 2.0, and $2.5 \mathrm{AU}$ are shown in figures 1,2 , and 3 , respectively. The upper line in each figure partially corrects for selection effects. Except for a flattening of the curve, which occurs around absolute magnitude 11 (particularly noticeable in fig. 2), the mass distributions given by Kessler (1969) are consistent with Dohnanyi's results. An upper limit to the spatial density of asteroids is given by Kessler (1968) by the requirement that the intensity of the counterglow is not exceeded by the total light reflected by the asteroids.

The cumulative asteroid spatial density $S$ at 2.5 AU (Kessler, 1969) is shown in figure 4 , along with the various models that have been discussed. Spherical particles with a mass density of $3.5 \mathrm{~g} / \mathrm{cm}^{3}$ and a geometric albedo of 0.1 were used. For a spatial density greater than $10^{-15}$ or $10^{-16}$ particles $/ \mathrm{m}^{3}$, the probability of encounter must be considered for large spacecraft (i.e., a 


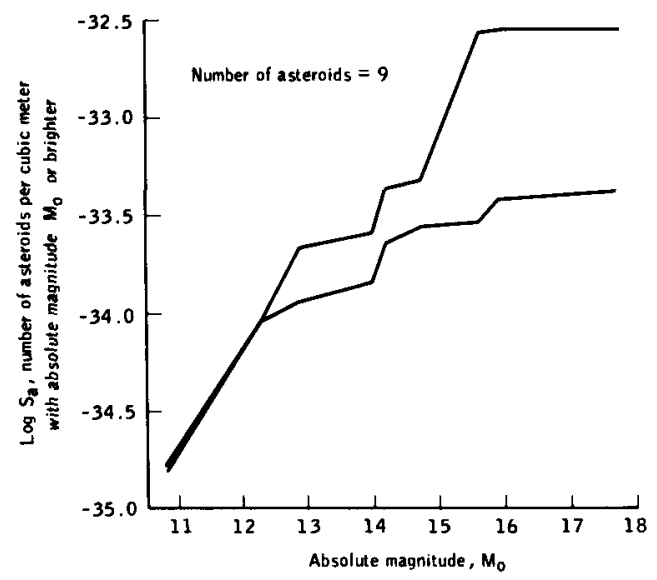

Figure 1.-Average asteroid spatial density in the ecliptic plane at 1.5 AU.

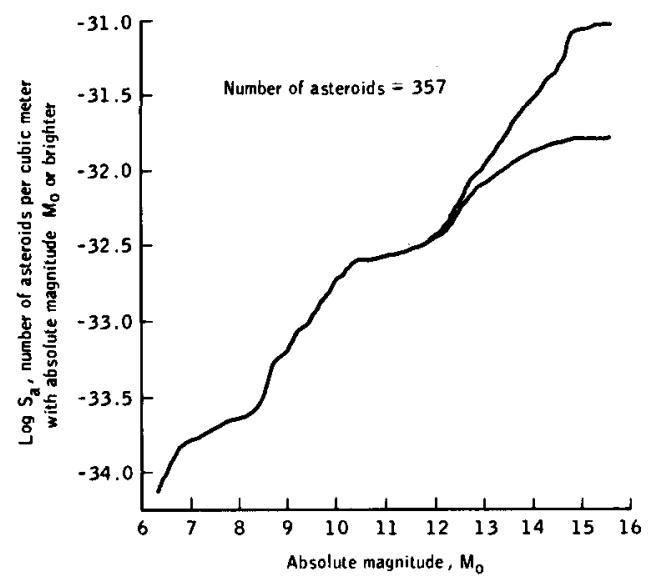

Figure 2.-Average asteroid spatial density in the ecliptic plane at $2.0 \mathrm{AU}$.

spacecraft with $500 \mathrm{~m}^{2}$ of surface area exposed to the environment for $1 \mathrm{yr}$ ). Therefore, if the asteroid spatial density varies as $m^{-1}$ (as suggested by Hawkins, 1960, and shown in fig. 4), such a spacecraft would require protection against impacts by masses as large as $10^{3} \mathrm{~g}$. However, this mass distribution exceeds the upper limit given by Kessler (1968) for masses less than $10^{4} \mathrm{~g}$. If the asteroid mass distribution varies as $\mathrm{m}^{-2 / 3}$ (Piotrowski, 1953), the spacecraft would have to be protected against asteroidal meteoroids of only $10^{-5} \mathrm{~g}$ (compared with cometary meteoroids of $10^{-3}$ or $10^{-2} \mathrm{~g}$ ).

The asteroid mass distribution suggested by Dohnanyi $(1969)\left(S \sim m^{-0.84}\right)$ gives an intermediate result that is consistent with the upper limit for asteroid 


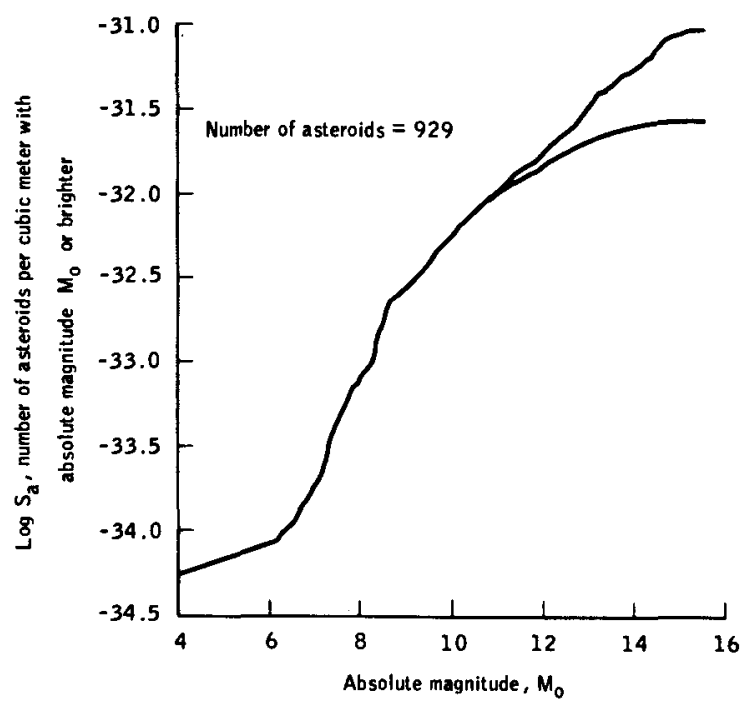

Figure 3.-Average asteroid spatial density in the ecliptic plane at $2.5 \mathrm{AU}$.

masses greater than $10^{-9}$. When Dohnanyi's mass distribution is extrapolated from the larger asteroids of Kessler (1969) at $R=1.0 \mathrm{AU}$, the resulting flux comes within a few percent of the flux found by Whipple (1967) from the Apollo asteroids, and the flux of meteoroids estimated from meteorite finds by Hawkins (1964) and Brown (1960). Such a mass law also gives results consistent with Öpik's prediction (Whipple, 1967) that the ratio of the number of Mars-crossing to Earth-crossing asteroids (of the same size) should be 300 .

Thus, this asteroid mass distribution will be adopted, and the assumption will be made that the mass distribution is independent of the distance from the Sun. The spatial density of asteroids with an absolute magnitude $M_{0}$ less than 10 can then be expressed as

$$
\log S_{a}=0.504 M_{0}+C_{R}
$$

where $S_{a}$ is the number of asteroids per cubic meter of absolute magnitude $M_{0}$ and brighter, and $C_{R}$ is a constant for each distance $R$ from the Sun.

The irregularity in the size distribution between asteroids with absolute magnitudes of 10 and 12 suggests that for $M_{0}>12, S_{a}$ in equation (1) should be reduced by a factor of 7.6 , or

$$
\log S_{a}=0.504 M_{0}+C_{R}-0.88
$$

The value of $C_{R}$ is then evaluated for each distance from the Sun by fitting equation (1) or (2) to the larger asteroids given by Kessler (1969); selection 


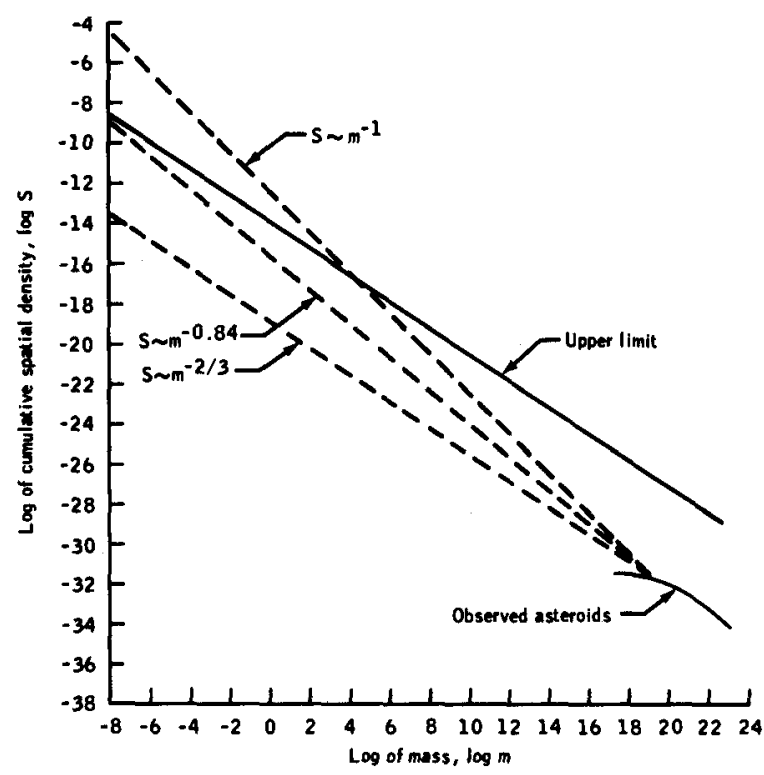

Figure 4.-Various asteroid mass-distribution models at $2.5 \mathrm{AU}$.

effects are minimal at the larger sizes. At $R=2.5 \mathrm{AU}, C_{2.5}=-37.18$. If a radial distribution is defined such that

$$
f(R)=C_{R}-C_{2.5}
$$

the spatial density in the ecliptic plane for the smaller asteroids becomes

$$
\log S_{a}=0.504 M_{0}-38.06+f(R)
$$

where $M_{0}>12$. The function $f(R)$ was evaluated from equation (3) and is given in figure 5 .

If the adopted values for asteroid mass density $\left(3.5 \mathrm{~g} / \mathrm{cm}^{3}\right)$ and geometric albedo (0.1) are used,

$$
\log S_{a}=0.84 \log m-15.79+f(R)
$$

where $10^{-9} \leqslant m \leqslant 10^{19}$, and $S_{a}$ is the number of asteroids per cubic meter near the ecliptic plane of mass $m$ (in grams) or larger at distance $R$ in astronomical units from the Sun. Equation (5) is limited to masses larger than $10^{-9} \mathrm{~g}$ for two reasons: (1) If the mass distribution is extrapolated to masses smaller than $10^{-9}$, the upper limit given by Kessler (1968) would be exceeded; and (2) solar radiation pressure and the Poynting-Robertson effect would 


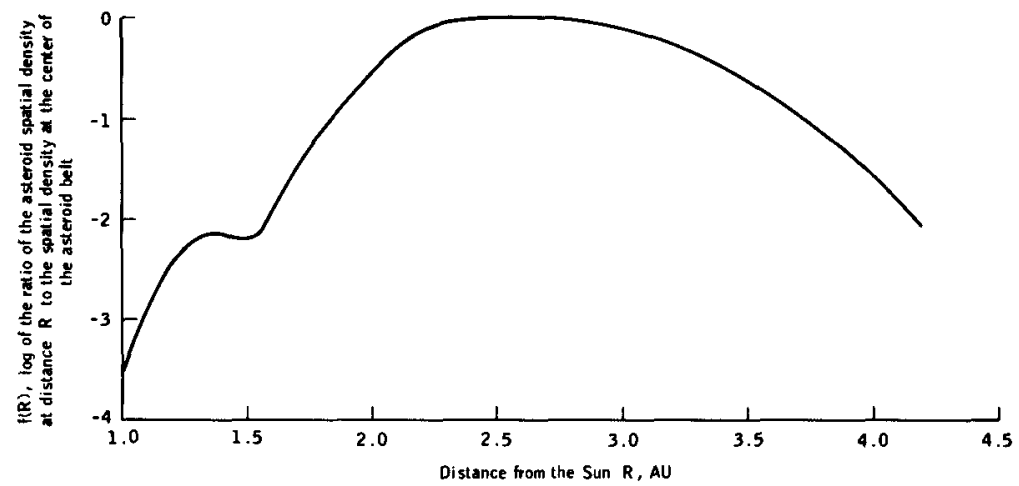

Figure 5.-Asteroid radial distribution.

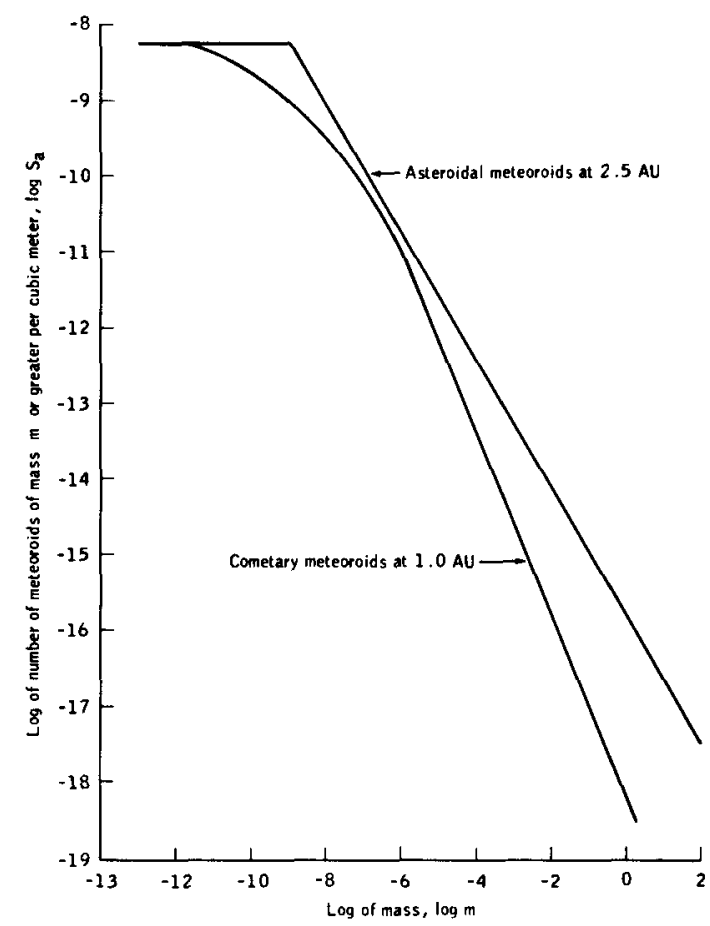

Figure 6.-Asteroid mass distribution compared to the cometary mass distribution near Earth.

probably cause the mass distribution between $10^{-12}$ and $10^{-6} \mathrm{~g}$ to change in a manner similar to the change in cometary meteoroids in the same mass range. Such a change can be approximated by limiting the distribution to asteroids of $10^{-9} \mathrm{~g}$ or larger. Use of a curved function similar to the function for cometary 
meteoroids would imply more knowledge of the asteroid mass distribution than is available. Equation (5) is compared with cometary meteoroids at $1 \mathrm{AU}$ in figure 6.

\section{LATITUDE AND LONGITUDE DEPENDENCE}

The asteroid spatial density is a function of both heliocentric latitude and longitude. At a latitude of $16^{\circ}$ from the ecliptic plane, the number of asteroids is reduced by about an order of magnitude from that given by equation (5). Between 1.5 and $2.4 \mathrm{AU}$, the spatial density is longitudinally dependent and reaches a maximum dependency at $R=1.8 \mathrm{AU}$. At $R=1.8 \mathrm{AU}$, the spatial density increases or decreases by one-half an order of magnitude in the direction of Jupiter's longitude of perihelion or the opposite direction, respectively. A complete discussion is contained in NASA SP-8038 (1970).

\section{RELATIVE VELOCITY}

The asteroids given in the 1967 Ephemeris volume were used to compute the average velocity of asteroids relative to a spacecraft. The asteroid data were corrected for observational selection effects (Kuiper et al., 1958), and weighted according to the probability of collision (Wetherill, 1967) with the spacecraft, and the velocity of each asteroid relative to the spacecraft was computed. Velocity distributions are obtained at distance $R$ from the Sun for a spacecraft whose velocity vector makes an angle $\theta$ with a circular orbit in the same plane. The speed of the spacecraft is $\sigma$, in units of the speed necessary to maintain a circular orbit of radius $R$ around the Sun. The average asteroid velocity $\vec{v}_{a}$ is. then found from each distribution. The velocity parameter $\bar{U}_{a}$ is introduced and is less dependent on distance from the Sun than is $\bar{v}_{a}$. The values of $\bar{U}_{a}$ shown in figure 7 were computed for $R=2.5 \mathrm{AU}$, but can be used with fair accuracy for all distances by applying the relationship

$$
\bar{v}_{a}=\bar{U}_{a} R^{-1 / 2}
$$

where $R$ is in astronomical units, $\bar{v}_{a}$ is in meters per second, and $\bar{U}_{a}$ is given in figure 7. A more detailed discussion, which introduces a slight $R$ dependence of $\bar{U}_{a}$, is contained in NASA SP-8038 (1970).

\section{FLUX AND NUMBER OF IMPACTS}

Flux on a randomly tumbling surface is related to spatial density and relative velocity by

$$
F_{a}=1 / 4 S_{a} v_{a}
$$

where $F_{a}$ is the number of asteroid impacts per square meter per second. The total number of impacts on a spacecraft is given by

$$
N_{a}=\int F_{a} A d t
$$




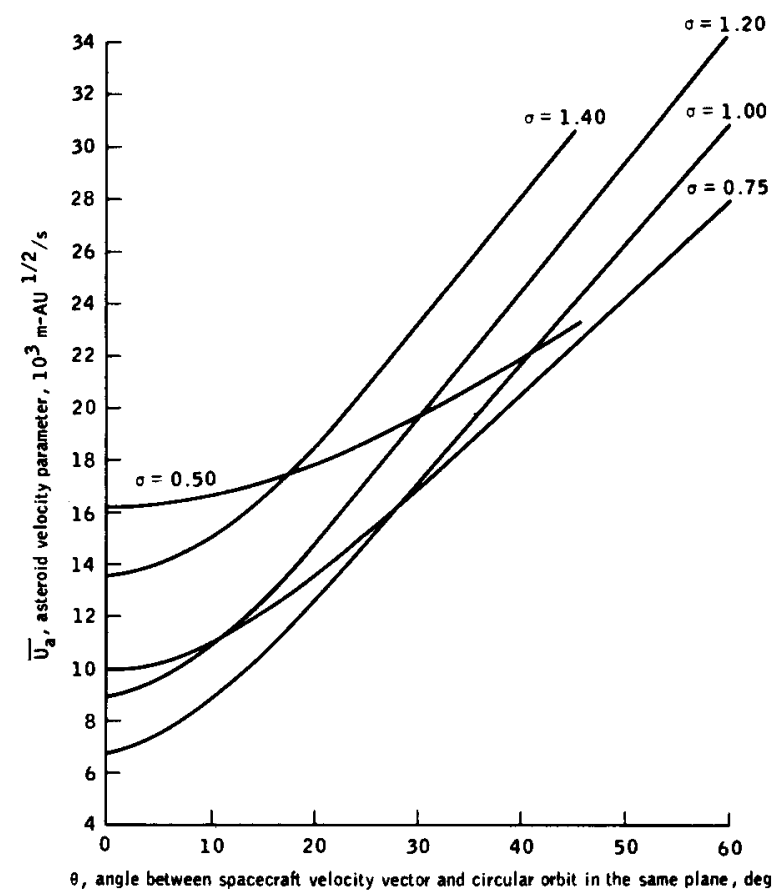

Figure 7.-Average relative velocity of asteroid particles at $R=2.5 \mathrm{AU}$ where $\sigma$ is the ratio of the heliocentric spacecraft speed to the speed of a spacecraft in a circular orbit at the same distance from the Sun.

where $A$ is the surface area of the spacecraft in square meters, $t$ is the time of spacecraft exposure to the environment, and the limits of integration are the beginning and end times of the mission.

\section{UNCERTAINTIES}

The model that describes the asteroid size distribution is anchored at the large-mass end by the observed asteroids and is assumed to vary as $m^{-\alpha}$. The assumption of the $m^{-\alpha}$ dependence and the uncertainty in the value of $\alpha$ probably constitute the largest uncertainties in the asteroidal-meteoroidenvironment model. An upper limit for the number of smaller asteroids is established by not allowing the number to be so great as to produce more reflected sunlight than is observed in the counterglow (Kessler, 1968). The value of $\alpha$ that was chosen for the model was not arbitrary, but (as was pointed out previously) was a value that agreed with the steady-state mass distribution based on collisions within the asteroid belt. The choice of a value for $\alpha$ is also consistent with a limitation in particle mass to $10^{-9} \mathrm{~g}$ and larger, again based on counterglow observations. A larger value for $\alpha$ than that selected for the 
model would lead to a particle cutoff at larger masses, to remain consistent with counterglow observations. Because no method exists for establishing the proper cutoff, the upper limit of the flux of particles with greater than a given mass is just the value that would not produce reflections exceeding that of the counterglow. The ratio of this upper limit to the model used (eq. (5)) is $26 m^{0.17}\left(m \geqslant 10^{-9} \mathrm{~g}\right)$. Thus, if the asteroid mass is limited to particles of $10^{-9} \mathrm{~g}$ or larger, the flux predicted from the model is essentially at the upper limit, whereas if the asteroid mass is limited to particles $1 \mathrm{~g}$ or larger, the limit of uncertainty permits the actual flux to be higher than the flux predicted by the model by a factor of 26 .

Additional uncertainty is introduced through the geometric albedo used in relating a particle distribution to the counterglow. Theoretically, geometric albedo may range from zero to infinity; however, the extremes of this range are never observed. A geometric albedo of 0.1 is used for estimating the mass of the visual asteroids in the asteroid-environment model. If the true albedo, however, is as low as 0.05 , the ratio of the upper limit of the asteroid flux predicted by the model becomes $52 \mathrm{~m}^{0.17}$.

The lower limit of the meteoroid population in the asteroid belt is set by the cometary environment. If the cometary meteoroid size distribution shown in figure 6 is assumed to vary as $R^{-3}$ (which is an extreme case), the ratio of the asteroid-model environment to the cometary environment in the heart of the asteroid belt is $4.7 \times 10^{3} \mathrm{~m}^{0.37}\left(m \geqslant 10^{-6}\right)$. This expression implies that the meteoroid flux in the asteroid belt could range from a factor of almost 30 lower than predicted by the asteroid model at $m=10^{-6} \mathrm{~g}$ to a factor of nearly 5000 lower for $m=1 \mathrm{~g}$.

\section{IMPACTS ON A SPACECRAFT}

If a typical trajectory through the asteroid belt (e.g., a mission to Jupiter) is integrated by using equations (8), (7), (6), and (5) and figures 5 and 7 , the total number of asteroid impacts per square meter of spacecraft surface area is given by

$$
\log \frac{N_{a}}{A}=-5.0-0.84 \log m
$$

The average asteroid velocity relative to the spacecraft would be approximately $15 \mathrm{~km} / \mathrm{s}$.

As can be seen from equation (9), the probability is small that a spacecraft with a random trajectory through the asteroid belt will collide with any of the large observed asteroids. In fact, only one asteroid of $10^{19} \mathrm{~g}$ or larger could be expected to pass within $10^{7} \mathrm{~km}$ of the spacecraft.

The real danger to spacecraft is from the much smaller asteroidal meteoroids. Equation (9) predicts an average of one asteroidal meteoroid impact of $10^{-6} \mathrm{~g}$ or larger for every square meter of spacecraft surface area. 
The upper limit for asteroidal particles of this size is about five impacts per square meter. Near the Earth, the flux of cometary meteoroids of $10^{-6} \mathrm{~g}$ or larger is about three impacts per square meter per year (NASA SP-8013, 1969), with an average velocity relative to the spacecraft of $20 \mathrm{~km} / \mathrm{s}$. Thus, for meteoroids in this size range, the asteroid belt is not likely to be more hazardous than the meteoroid environment near Earth.

However, assume that it is desired to insure that a spacecraft having $100 \mathrm{~m}^{2}$ of surface area has at least a 0.99 probability of not being penetrated by a meteoroid. The spacecraft must then be designed to withstand (from eq. (9)) an impact by a particle of $10^{-1.2} \mathrm{~g}$ with a relative velocity of $15 \mathrm{~km} / \mathrm{s}$. (This compares with a cometary particle mass of $10^{-2.4} \mathrm{~g}$ for a similar spacecraft near Earth for $1 \mathrm{yr}$.) If the spacecraft were designed to withstand an impact from a $10^{-1.2} \mathrm{~g}$ meteoroid (which could add hundreds of kilograms to the spacecraft weight), and the upper limit to the asteroid flux were encountered, then the probability of no penetration would be reduced to 0.72 . To design a spacecraft using the upper limit would require protection against a $10 \mathrm{~g}$ asteroidal meteoroid, which would severely increase the weight of the spacecraft.

\section{REFERENCES}

Baldwin, Ralph B. 1964, Lunar Crater Counts. Astron. J. 69(5), 377-392.

Brown, Harrison. 1960, The Density and Mass Distribution of Meteoritic Bodies in the Neighborhood of the Earth's Orbit. J. Geophys. Res. 65(6), 1679-1683.

Dohnanyi, J. S. 1969, Collisional Model of Asteroids and Their Debris. J. Geophys. Res. 74(10), 2531-2554.

Hartmann, William K. 1965, Secular Changes in Meteoritic Flux Through the History of the Solar System. Icarus 4(2), 207-213.

Hartmann, William K. 1968, Lunar Crater Counts-VI: The Young Craters Tycho, Aristarchus, and Copernicus. Communications of the Lunar and Planetary Lab., vol. 7, no. 119, pp. 145-156. Univ. of Arizona Press. Tucson.

Hawkins, Gerald S. 1960, Asteroidal Fragments. Astron. J. 65(5), 318-322.

Hawkins, G. S. 1964, Interplanetary Debris Near the Earth. Ann. Rev. Astron. Astrophys. 2, 149-164.

Kessler, Donald J. 1968, Upper Limit on the Spatial Density of Asteroidal Debris. AIAA J. 6(12), 2450.

Kessler, D. J. 1969, Spatial Density of the Known Asteroids in the Ecliptic Plane. NASA TM X-58026.

Kuiper, G. P., Fujita, Y., Gehrels, T., Groeneveld, I., Kent, J., Van Biesbroeck, G., and Houten, C. J. van. 1958, Survey of Asteroids. Astrophys. J. Suppl. Ser. 32, vol. III, pp. 289-335.

Marcus, A. H. 1966, A Stochastic Model for the Formation and Survival of Lunar Craters. Icarus 5, 165-200.

Marcus, A. H. 1968, Number Density of Martian Craters. Bellcomm Rept. TR-68-710-1.

NASA SP-8013. 1969, Meteoroid Environment Model-1969 (Near Earth to Lunar Surface).

NASA SP-8038. 1970, Meteoroid Environment Model-1970 (Interplanetary and Planetary).

Piotrowski, S. 1953, Collisions of Asteroids. Acta Astron. 5, (Oct.), 115-138.

Wetherill, G. W. 1967, Collisions in the Asteroid Belt. J. Geophys. Res. 72(9), 2429-2444. 
Whipple, Fred L. 1967, On Maintaining the Meteoritic Complex. Smithson. Astrophys. Observ. Special Rept. 239. (Also available in NASA SP-150, 1967, pp. 409-426.)

\section{DISCUSSION}

ALFVÉN: Have you made any estimates on how much danger a space mission to a comet might have, considering the fact that comets are associated with meteor streams?

KESSLER: No. 